\title{
Dose reduction in whole-body computed tomography of multiple injuries (DoReMl): protocol for a prospective cohort study
}

Dirk Stengel ${ }^{1,2,4^{*}+}$, Caspar Ottersbach ${ }^{1 \dagger}$, Thomas Kahl $^{3}$, Constanze Nikulka $^{3}$, Claas Güthoff ${ }^{1}$, Thomas Hartel ${ }^{2}$, Sophia Hünnebeck ${ }^{1,2}$, Axel Ekkernkamp ${ }^{2}$ and Sven Mutze ${ }^{3}$

\begin{abstract}
Background: Single-pass, contrast-enhanced whole body multidetector computed tomography (MDCT) emerged as the diagnostic standard for evaluating patients with major trauma. Modern iterative image algorithms showed high image quality at a much lower radiation dose in the non-trauma setting. This study aims at investigating whether the radiation dose can safely be reduced in trauma patients without compromising the diagnostic accuracy and image quality.

Methods/Design: Prospective observational study with two consecutive cohorts of patients. Setting: A high-volume, academic, supra-regional trauma centre in Germany.

Study population: Consecutive male and female patients who 1. had been exposed to a high-velocity trauma mechanism, 2. present with clinical evidence or high suspicion of multiple trauma (predicted Injury Severity Score [ISS] 216) and 3. are scheduled for primary MDCT based on the decision of the trauma leader on call. Imaging protocols: In a before/after design, a consecutive series of 500 patients will undergo single-pass, whole-body 128-row multi-detector computed tomography (MDCT) with a standard, as low as possible radiation dose. This will be followed by a consecutive series of 500 patients undergoing an approved ultra-low dose MDCT protocol using an image processing algorithm.

Data: Routine administrative data and electronic patient records, as well as digital images stored in a picture archiving and communications system will serve as the primary data source. The protocol was approved by the institutional review board.

Main outcomes: (1) incidence of delayed diagnoses, (2) diagnostic accuracy, as correlated to the reference standard of a synopsis of all subsequent clinical, imaging, surgical and autopsy findings, (3) patients' safety, (4) radiation exposure (e.g. effective dose), (5) subjective image quality (assessed independently radiologists and trauma surgeons on a 100-mm visual analogue scale), (6) objective image quality (e.g., contrast-to-noise ratio).

Analysis: Multivariate regression will be employed to adjust and correct the findings for time and cohort effects. An exploratory interim analysis halfway after introduction of low-dose MDCT will be conducted to assess whether this protocol is clearly inferior or superior to the current standard.
\end{abstract}

Discussion: Although non-experimental, this study will generate first large-scale data on the utility of imaging-enhancing algorithms in whole-body MDCT for major blunt trauma.

Trial registration: Current Controlled Trials ISRCTN74557102.

\footnotetext{
* Correspondence: dirk.stengel@ukb.de

${ }^{\dagger}$ Equal contributors

${ }^{1}$ Centre for Clinical Research, Unfallkrankenhaus Berlin Trauma Centre,

Warener Str. 7, Berlin 12683, Germany

${ }^{2}$ Department of Trauma and Orthopaedic Surgery, Unfallkrankenhaus Berlin

Trauma Centre, Warener Str. 7, Berlin 12683, Germany

Full list of author information is available at the end of the article
} 


\section{Background}

According to the most recent injury statistics, every two minutes someone dies of an injury in the European Union [1]. Trauma is, and will remain a significant contributor to years of productive life lost, disability, and health care service costs worldwide [2-4].

The survival and functional prognosis of patients who suffered severe and multiple injuries improved significantly in the industrialized countries [5-8]. Time to appropriate care is an accepted predictor of trauma outcomes, and transport, triage, and work-up intervals must respect this decisive factor. Also, immediately life-threatening injuries and sources of bleeding must be identified (or excluded) with high accuracy $[9,10]$. Apart from damage-control resuscitation and surgery, the introduction of contrastenhanced, whole-body, multi-detector computed tomography may be regarded as one of the most important interventions which changed the face of trauma care during the past decade [11-14].

In a recent survey of UK emergency departments, 41 out of $184(22.3 \%)$ respondents indicated that they had a panscan policy for major trauma patients, with marked regional variations in availability and reporting times [15]. Data from the German TraumaRegister ${ }^{\mathrm{D}}$ suggest that incorporating a routine pan-scan into trauma resuscitation may increase the ratio of observed to expected survivors [16]. A recent analysis confirmed the results in a subsequent cohort and unstable patients [17]. Yet, many injuries to solid organs (e.g., brain, liver, spleen) demarcate only after sufficient resuscitation with crystalloid fluids and blood products. We recently demonstrated in a large cohort of 982 patients that an initial pan-scan is capable to prove but not to exclude injuries to various body regions [18].

Opponents of the whole-body scan have been voicing concerns about patients' overexposure to radiation with the increasing and often uncritical use of this technology [19-24].

Exposure to radiation for diagnostic purposes is an emerging ethical, medico-legal and public-health issue [25]. It has been estimated that, with the widespread and liberal use of CT, $1.5-2.0 \%$ of all cancers in the US may now be attributable to the radiation from CT examinations [21]. The median lifetime attributable risk (LAR) of cancer is 4.0 (IQR $0.8-11.1$ ) / 1000 for a multiphase abdomen and pelvis CT scan [26].

Modern 64-, 128- and 256-row MDCT scanners are regarded to produce lower dose indexes $\left(\mathrm{CTDI}_{\mathrm{vol}}\right)$ than earlier generation single- or 4-row hardware. However, Harrieder et al. recently demonstrated in a retrospective study that, because of enhanced scan lengths, the doselength-product (DLP) may remain similar or even increase with modern hardware [27]. The authors, however, provided little information on patient demographics (e.g., BMI, ISS) and did not only compare different multi-detector-row technologies but also scanners made by different manufacturers. A comparison of 64-row scanners of four leading companies suggested highest patient mean effective doses (in $\mathrm{mSv}$ ) produced by the GE LightSpeed VCT $(12.7 \pm 2.6)$, followed by the Toshiba Aquilion (11.1 \pm 3.3$)$, Philips Brilliance (9.5 \pm 0.4$)$ and Siemens Somatom (9.1 \pm 1.1$)$ [28].

So-called adaptive statistical iterative algorithms (ASIR), derived from a Bayesian framework, incorporate i. random fluctuations in sinogram measurements [29], ii. nonideality, iii. different degrees of data credibility, iv. a priori information about the distribution of the image space, and other previously unconsidered variables to enhance resolution and reduce artefacts and noise [30].

Distinct technologies like Veo (Model Based Image Reconstruction, GE Healthcare) or IRIS (Iterative Reconstruction in Image Space, Siemens) received FDA approval [31].

Apart from phantom studies and smaller case series, there is currently sparse evidence from head-to-head studies about the effect of ASIR technology on objective and subjective image quality, and exposure to radiation. Exemplarily, Prakash et al. from Boston showed in a cohort of 222 patients who had previously undergone abdominal CT with filtered back projection (FBP) technology and were scheduled for follow-up ASIR scan that CTDL vol $_{\text {and DLP }}$ were markedly reduced without compromising image quality [32]. The Prakash group showed similar results in chest CT $[33,34]$. Other investigators reported similar dose reductions with similar image quality in CT portovenography [35], cerebral CT [36], and abdomino-pelvic CT scans [37].

There is evidence from phantom tests and rigorous clinical evaluations with global clinical collaborators which demonstrate the potential of the FDA-approved iDose system (Philips Healthcare, Eindhoven, The Netherlands) to improve image quality and / or lower radiation dose levels beyond those previously achievable with conventional, routine-dose acquisitions, or FBP reconstructions [38-43].

The combination of BMI-adapted protocols with iterative reconstruction algorithms can reduce radiation exposure to patients and simultaneously improve image quality [38].

Previous non-trauma / elective CT investigations suggest that iterative reconstruction algorithms may maintain image quality at a much lower radiation dose. Yet, large-scale, prospective evidence on the safety and effectiveness of low-dose CT in trauma patients is still sparse. A single retrospective study investigated possible dose reduction at maintained image quality in subjects with multiple injuries [44]. Since results were not adjusted for baseline imbalances (e.g., a 7\% difference in the prevalence of comorbidity), conclusions and inferences from this study are difficult to interpret.

Patients with multiple injuries are typically young and thus at a higher risk of CT-induced cancer. Reducing exposure to radiation with constant image quality and diagnostic accuracy in this vulnerable cohort is a key 
goal of trauma services worldwide, and evidence whether imaging at lower doses of radiation is feasible, safe, and effective is needed. On the other hand, it may be argued that, given the proven benefit of MDCT in lowering trauma-associated mortality, the primary scan must exhaust the full potential of the method and should aim for maximum quality of images to avoid false-negative results, regardless of exposure to radiation.

\section{Methods/Design \\ Design}

The Dose Reduction in whole-body computed tomography of Multiple Injuries (DoReMI) investigation is a prospective cohort study employing two large, consecutive cohorts of patients undergoing different approved and established MDCT protocols. All data will be recorded, stored and processed under the conditions of daily practice of trauma care. Gathered data will form the basis for a later interrupted time-series comparison between the effectiveness and safety of the scanning protocols.

\section{Design history and IRB concerns}

The study was originally planned as non-inferiority randomized controlled trial (NI-RCT) with the following hypotheses:

Null hypothesis $\mathrm{H}_{0}$ notated as:

$$
\mathrm{H}_{0}: \mathrm{C}-\mathrm{T} \geq \mathrm{M}(\mathrm{C} \text { is superior to } \mathrm{T})
$$

where $\mathrm{C}=$ image quality in the standard-of-care arm, $\mathrm{T}=$ image quality in the experimental (iDose) arm, and $\mathrm{M}=$ non-inferiority margin

Alternative hypothesis $\mathrm{H}_{\mathrm{A}}$ expressed as

$$
\mathrm{H}_{\mathrm{A}}: \mathrm{C}-\mathrm{T}<\mathrm{M}(\mathrm{T} \text { is not inferior to } \mathrm{C})
$$

In clinical terms, the primary hypothesis read as:

In patients with multiple trauma scheduled for singlepass, contrast-enhanced whole-body 128-row MDCT, the iDose algorithm will generate a subjective image quality rated on a visual analogue scale $(0-100 \mathrm{~mm})$ which is not inferior to standard image production and processing.

The non-inferiority margin (NIM) was set at $1 \mathrm{~mm}$, with a standard deviation of $4 \mathrm{~mm}$ (derived from a pilot study of $2 \times 20$ patients). The sample size for a noninferiority trial of two means was computed using PASS 11.0 software (NCSS, LLC. Kaysville, Utah, USA). For a fixed NIM of $1 \mathrm{~mm}$, the needed sample size per group was 253 patients to confirm non-inferiority of the experimental compared to the control intervention with a one-sided alpha 0.025 and a power of $80 \%$.

The protocol was submitted to the institutional review board (IRB) of the Charité University Medical Centre, Berlin, Germany, in May 2013. It accounted for 1. the inclusion of unconscious and minor patients in an emergency setting (with distinct processes of informing relatives and deferred informed consent procedures) and 2. varying exposure to radiation for diagnostic purposes as the intervention of interest. Given that MDCT trauma imaging at a lower than usual radiation dose is not the current standard of care in Germany, the institutional review board issued concerns that patients in the experimental group are at risk of missed injuries (not that patients in the standard-of-care group may be exposed to a higher than needed effective dose). Consequently, the initial proposal was rejected. A revised proposal submitted in June 2013 could not solve the IRB's concerns.

Consequently, the investigators changed their approach to comply with the IRB's recommendations, and consented on a before/after cohort study. All patients in this study will receive an accepted diagnostic standard of care using approved technologies but will not undergo random allocation to either modality. One may still argue that injuries are acute, unforeseeable events, and there remains a random component in the study set-up.

The ultimate consequence of shifting from an experimental to an observational design was the increase in the sample size to allow for robust multivariate modelling and adjustment of results for confounding.

\section{Objectives}

The following objectives will be addressed:

1. To determine the incidence of missed or delayed diagnoses with either imaging scheme,

2. To define the diagnostic accuracy of either MDCT protocol, based on the synopsis of clinical, imaging, and interventional information gathered until discharge,

3. To evaluate patients' safety with either protocol,

4. To determine the exposure to radiation and the LAR of cancer with either approach,

5. To evaluate subjective and objective measures of image quality.

\section{Setting}

This is a single-centre non-interventional study using approved and established MDCT imaging protocols for severely injured patients. The study compares a cohort of subjects receiving the standard of care, i.e., single-pass, whole-body 128-MDCT with regular image acquisition and processing, with a subsequent cohort receiving singlepass, whole-body 128-MDCT with a radiation-dose reducing iterative reconstruction method (iDose). Table 1 summarizes key items of both protocols. Figure 1 shows the anticipated study flow.

\section{Primary outcome}

Delayed diagnoses comprise pathological anatomic and / or physiologic findings which demand clinical awareness, 
Table 1 Key scanning parameters in the experimental and the control group

\begin{tabular}{lcc}
\hline & $\begin{array}{c}\text { Standard dose } \\
\text { 128-row MDCT } \\
\text { pan-scan }\end{array}$ & $\begin{array}{c}\text { Low dose } \\
\text { 128-row MDCT } \\
\text { pan-scan + iDose }\end{array}$ \\
\hline $\begin{array}{lcc}\text { Detector z } \\
\text { coverage, mm }\end{array}$ & 40 \\
kVp & 120 & 120 \\
Exposure, mAs & 180 & 90 \\
iDose Level & off & $4(6)$ \\
Recon filter & B & B (C) \\
CTDI, mGy & 11.9 & 5.9 \\
\hline
\end{tabular}

or, in case of therapeutically relevant pathological results, should prompt immediate therapeutic action.

We will estimate the incidence of delayed diagnoses based on a synopsis of all subsequent clinical, imaging, intra-operative, and autopsy findings until discharge. We will further distinguish between clinically relevant and all diagnoses. Relevant diagnoses are defined as severe injuries suiting an Abbreviated Injury Scale [AIS] score $\geq 3$ and/or injuries demanding surgical and/or trans-vascular intervention. Non-traumatic incidental findings like tumours or tumour-like lesions demanding specific work-up and/or treatment will be recorded in an exploratory attempt.

\section{Secondary outcomes \\ Accuracy}

Accuracy (or diagnostic efficacy) is a key indicator of performance of an imaging technology. In pan-CT for trauma, there are five variables contributing to overall accuracy:

1. The general technical capability of CT technology to depict a certain pathology (resolution)

2. Trauma-associated, acutely impaired physiological parameters (e.g., hypotension, haemorrhagic shock, centralized circulation) which hamper organ perfusion and/or vessel contrast

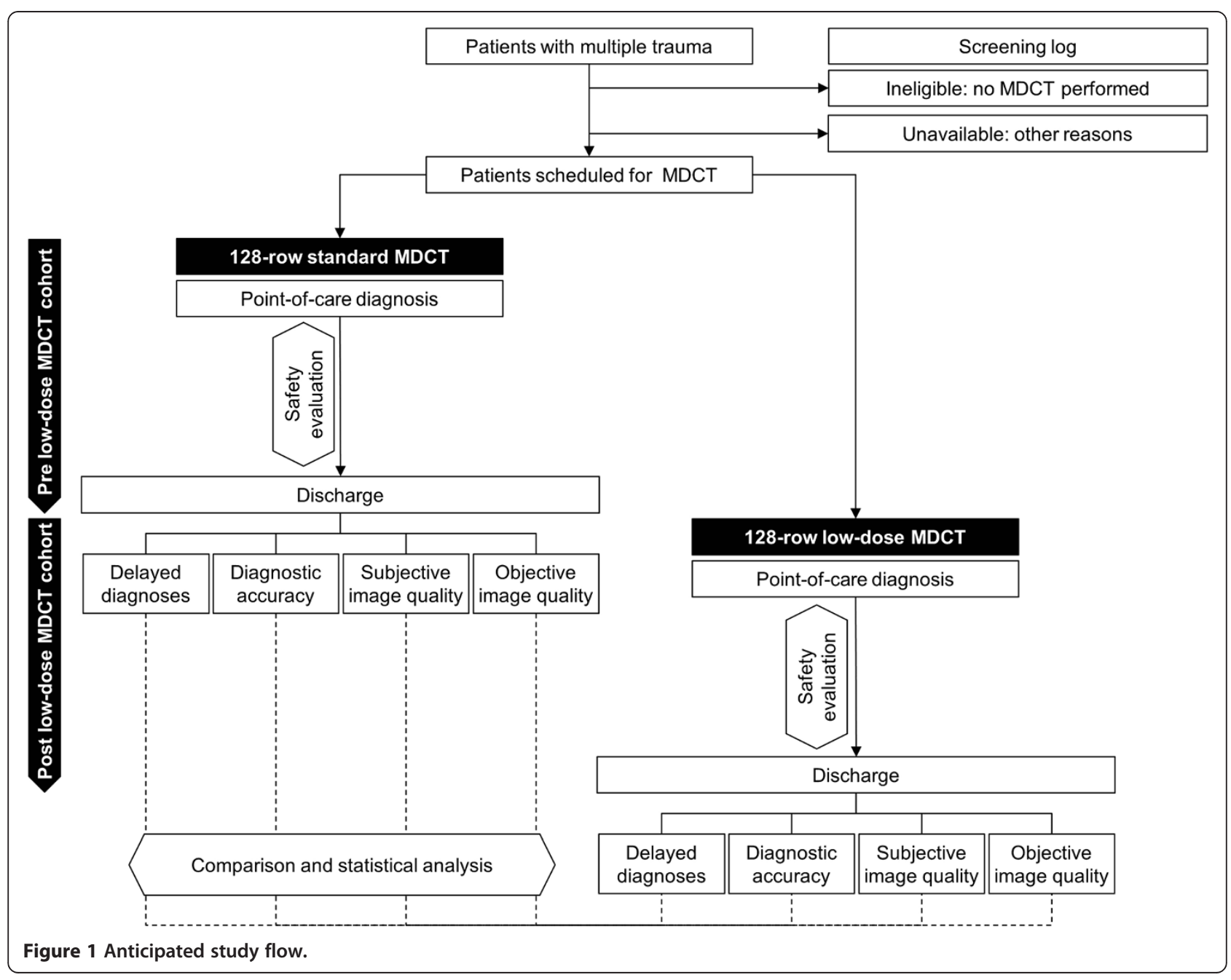


3. The interval needed for tissue contusions and hematomas to demark or expand

4. Interpretation of images by the radiologist on call

5. The severity and/or therapeutic consequences of findings

In addition to the incidence of delayed diagnoses, we will compute standard indicators of diagnostic test accuracy (i.e., sensitivity, specificity, positive and negative predictive values, areas under the ROC). Different models will be created for various endpoints (e.g., all missed injuries, therapeutically relevant findings, and others).

\section{Safety}

Any situation in which the individual patient's safety is assumed to be compromised will be recorded and evaluated in a cumulative fashion. Safety-relevant events will be entered in the local Critical Incident Reporting System (CIRS), hosted by the Medical Council (Ärztekammer) of Berlin, Germany. Since this is an observational study, we will use common definitions of adverse events (AE), serious adverse events (SAE), and Suspected Unexpected Serious Adverse Reactions (SUSAR), as outlined in $\mathrm{ICH}$ Topic E2B (Clinical Safety Data Management: Data Elements for Transmission of Individual Case Safety Reports), and most recently in the amended and updated DIN-ISO 14155 guideline, but do not implement a regular reporting procedure.

\section{Radiation dose and estimated LAR of cancer}

Dose calculation includes

- CTDI ${ }_{\text {vol }}$, scan length, DLP as reported by the scanner (dose report page in PACS), and verified by software CT-Expo, if values are ambiguous or information is missing

- Effective dose (in mSv) calculated by CT-Expo from documented parameters, based on most recent ICRP recommendations (modified tissue-weighting factors)

\section{Subjective image quality}

Numerous approaches have been proposed to quantify the quality of CT images in the transversal plane, and to generate resource-intensive but clinically required coronal, sagittal, and 3-D reconstruction views.

Basically, there are two different, still complementary concepts to quantify image quality:

1. Subjective evaluation of quality, ideally assessed by two or more independent expert raters in a random, blinded (towards the underlying processing algorithm) fashion, using pre-defined body areas and instruments or scales
2. Objective evaluation, using physical parameters like contrast/signal, noise, and the ratio of both

There is currently no consensus or a reproducible pattern of criteria available to distinguish "good" or "diagnostic" from "unacceptable" or "un-diagnostic" images. In 1999, the European Commission had summarized quality criteria in a guideline (EUR $16262 \mathrm{EN}$, http://w3.tue.nl/fileadmin/ sbd/Documenten/Leergang/BSM/European_Guidelines_ Quality_Criteria_Computed_Tomography_Eur_16252.pdf) which had lastly been updated in 2004 (http://www.msct. eu/CT_Quality_Criteria.htm).

Latest updates on the web-platform of the Imaging Performance Assessment of CT Scanners (IMPACT, http:// www.impactscan.org/) working group have been made in 2010.

From a statistical point of view, 3- and 5-point ordinal scales have the disadvantage of a non-normal / nonparametric distribution. Although frequently used, the central measure of these scales is not the parametric mean but the geometric mean or median. For sample size calculations and full-scale variance, a visual analogue scale is the better option to determine image quality.

To overcome the limitations with established rating scales, wet set up a pilot study using $2 \times 20$ sets of images from patients with multiple trauma. The control set consisted of images obtained by a 64-row MDCT scanner (Brilliance 64, Philips, Germany), with scanning parameters published elsewhere [18]. The experimental set comprised images obtained by the 128-row MDCT (Ingenuity Core 128, Philips) scanner to be used in the planned project.

Two independent radiologists blinded to the scanner generation and protocol rated image quality on a 100$\mathrm{mm}$ visual analogue scale, focusing on lung, bone, vessel, fluid/fat and organ tissue contrast.

The arithmetic mean of both raters with the pooled standard deviation s, calculated as

$$
\mathrm{s}=\sqrt{\frac{\left(\mathrm{n}_{1}-1\right) \mathrm{S}_{1}^{2}+\left(\mathrm{n}_{2}-1\right) \mathrm{S}_{2}^{2}}{\mathrm{n}_{1}+\mathrm{n}_{2}-2}}
$$

was used for quantitative assessment of differences between groups. Here, $n_{1}$ and $n_{2}$ are the number patients / images assessed by rater 1 and 2 , and $S_{1}$ and $S_{2}$ are the standard deviations of means.

There were no marked differences in quality ratings between images obtained by either method (Table 2). According to the observed standard deviations, a $1 \pm$ $4 \mathrm{~mm}$ difference on the 100-mm VAS appears as the minimally detectable difference between both imaging protocols. 
Table 2 Summary of the results of the pilot study

\begin{tabular}{lccc}
\hline Tissue & \multicolumn{2}{c}{ Pooled mean (SD) } & Mean difference $(\mathbf{9 5 \%} \mathbf{C l})$ \\
\cline { 2 - 3 } & $\mathbf{6 4}$-row & 128-row & \\
\hline Lung & $93.90(1.89)$ & $93.30(2.03)$ & $0.60(-0.66$ to 1.86$)$ \\
Bone & $93.28(2.32)$ & $92.15(3.63)$ & $1.13(-0.82$ to 3.08$)$ \\
Vessel & $91.60(3.31)$ & $89.90(4.84)$ & $1.70(-0.95$ to 4.35$)$ \\
Organ & $89.60(3.92)$ & $85.98(7.87)$ & $3.62(-0.36$ to 7.60$)$ \\
Fluid/Fat & $92.98(3.39)$ & $90.78(4.43)$ & $2.20(-0.33$ to 4.73$)$
\end{tabular}

A 100-mm VAS was used for subjective image quality assessment.

\section{Objective image quality}

Contrast and noise, and the ratio of both, are amongst the most influential indices to describe the objective quality of CT images.

The contrast-to-noise ratio (CNR) is defined as

$$
\mathrm{CNR}=\frac{\left|\mathrm{s}_{1}-\mathrm{s}_{2}\right|}{\sqrt{\frac{\sigma_{1}^{2}+\sigma_{2}^{2}}{2}}}
$$

where $\mathrm{S}_{1}=$ mean $\mathrm{CT}$ number (in $\mathrm{HU}$ ) in region of interest $1\left(\mathrm{ROI}_{1}\right) 1, \mathrm{~S}_{2}=$ mean $\mathrm{HU}$ in $\mathrm{ROI}_{2}, \sigma_{1}=$ mean $\mathrm{SD}$ of the mean $\mathrm{HU}$ in $\mathrm{ROI}_{1}$, and $\sigma_{2}$ mean $\mathrm{SD}$ of the mean $\mathrm{HU}$ in $\mathrm{ROI}_{2}$

Standard ROIs of similar size and position are placed in prominent anatomical structures such as the aorta, liver, other solid organs and spinal muscles. If metal implants are present, then ROIs shall also be placed in the vicinity.

\section{Patients}

This study will include a representative German and European trauma population, meaning that eligible patients had

1. sustained blunt major trauma by a car crash or fall from a height

2. been resuscitated on scene by a multi-professional team of paramedics and emergency physicians, including sedation / general anaesthesia and airway management by oro-tracheal intubation

Thus, the target sample unavoidably includes incompetent (i.e., unconscious and ventilated, probably hemodynamically unstable) patients.

An injury is an unpredictable event that may occur in all age groups, and regardless of other medical conditions. Some patients may also not have reached the legal age of consent at the time of injury.

Patients with the following characteristics are eligible to be enrolled in the planned study:

1. Male and female patients of all ages

2. Suspected or already proven blunt multiple trauma, as indicated by the presence of injuries to $\geq 2$ body regions which, alone or in combination, are lifethreatening, or an ISS $\geq 16$

3. Indication for primary, single-pass whole-body CT by judgment of the trauma leader in charge and according to red flag criteria outlined in the German Trauma Association's clinical practice guideline on the management of multiple trauma [45]:

- any injury mechanism exposing patients to a high risk of multiple trauma (i.e., road traffic crash with presumed high-energy trauma like extrication or death of a car occupant, pedestrian struck by a vehicle, fall from height)

- need for technical rescue

- impaired physical or physiological status (i.e., unconsciousness, intubation and ventilation, obvious signs of injury like bruises, haematoma, open wounds or fractures, hemodynamic instability)

- suspicion of severe trauma confirmed by paramedics and/or emergency doctors on scene

This study evaluates routine clinical practice and consecutive patients. Since this includes only subjects who undergo primary, single-pass MDCT for clinical indication, those

1. considered unsuitable for CT for any reason (e.g., need for immediate life-saving thoracotomy, laparotomy, or cranial trepanation)

2. with futile resuscitation and / or CPR efforts

3. declared dead on arrival will be missed by this study.

\section{Recruitment and documentation}

Patients will be recruited under routine clinical care conditions, and documentation will mainly comprise data collected for administrative issues or routinely entered in the electronic patient chart. Also, all radiological images will be stored in a PACS for later processing and evaluation.

We will record all patients participating in this study immediately after they underwent the MDCT scan. Informed consent will be gathered by relatives or the next of kin, or individual patients as soon as they are able to consent in using their data for scientific purposes.

This study focuses on delayed and missed injuries, and the accuracy of the primary MDCT scan. Thus, it needs a diagnostic reference or gold standard.

The probably best reference method to which initial MDCT findings can be compared is the clinical and radiological follow-up. If the initial MDCT misses injuries in severely injured patients, it is very likely they will be detected during hospital stay (the same holds true for suspected pathological findings which cannot be confirmed or verified on follow-up imaging). 
This study will employ a synopsis of all clinical (including intra-operative) and radiological findings collected until discharge as the diagnostic reference standard. All these items will be traced from routinely available information.

\section{Sample size considerations and calculations}

The sample size is calculated to guarantee a certain precision of a range of estimates, not to achieve a certain power to detect an a priori defined effect size.

With 500 eligible cases in either cohort, this study fulfils the following prerequisites:

1. $95 \%$ confidence intervals of both risk and mean differences must not exceed the point estimate by more than $5 \%$ in either direction

2. multivariate modelling must be possible for five independent covariates (respecting Harrell's rule)

\section{Analysis plan}

Accepted statistical methods will be used to determine whether the distribution of baseline variables and outcome results are compatible with chance. In general, results will be presented as means, medians, or proportions, and differences in means and proportions, including appropriate measures of distribution and 95\% confidence intervals.

We will use established methods to model observational, specifically time-series data, stratified for the different imaging protocols and potential confounding, independent variables. This will include multi-variable generalized linear, random- and mixed-effects regression analyses.

Missing values will be addressed by adequate methods (including multiple imputation techniques).

Multivariate modelling will always be conducted using sound selection, entry, and exclusion of variables, and after testing for model fit and plausibility.

EQUATOR-, GCP-, GEP-, and AMA-conformity will be assured when presenting results. Raw numbers and counts will be provided together with percentages. The STATA 11.0 software package will be employed for all analyses.

\section{Data management and quality assurance}

Data management, including data entry, plausibility checks and query generation will be performed by the local trial coordinating unit (Centre of Clinical Research, Unfallkrankenhaus Berlin).

Subjective quality of images, as assessed on 100-mm VAS for general impression and distinct quality of structures, tissues and sites specified earlier, will be graded by two radiologists in a paper-based fashion. Tick-marks on the VAS will be measured and be entered in an electronic data capture (EDC) system by appointed research assistants at the trial unit.
All further data will be handled and stored electronically for further processing, and processed in the database. Data handling and protection will conducted according to GCP and federal laws of data protection.

All materials pertaining to the investigation will be documented by research staff of the trial unit, sorted and kept in closed archives. The investigator should maintain all source data together with related study documentation for the maximum period of time permitted by the hospital, institution or private practice, but not less than that minimally prescribed by the local authorities after the clinical part of the study has been completed.

\section{Discussion}

This is the first study which assesses the potential value of a dose-reducing MDCT regimen in patients with multiple and severe trauma. The investigators initially planned a RCT which was rejected by the local IRB.

The current protocol aims at balancing regulatory and methodological issues. While the present study (because of its non-experimental design) cannot formally prove that low-dose MDCT is feasible, safe, and evenly accurate compared to normal-dose MDCT in the trauma setting, the cohort size, the setting, and the anticipated characteristics of patient cohorts will guarantee both high internal and external validity of findings. Advanced statistical approaches will allow for quasi-confirmatory inferences. In the absence of large-scales RCTs, this study may provide the best available evidence to decide in favour of one or the other diagnostic approach.

\section{Competing interests}

This is an investigator-initiated study, and no external funding by commercial parties was received for planning this research project. The authors declare they have no competing interest.

\section{Authors' contributions}

SM and DS conceived the study, and worked together in adapting the protocol according to IRB recommendations and obligations. DS drafted the initial and further protocol versions and developed the initial and subsequent investigation plans. CG is the data manager and statistician of this study, and set up data entry forms and the electronic data capture system. $\mathrm{CO}$ is responsible for study administration and regulatory affairs. $\mathrm{TK}, \mathrm{CN}, \mathrm{TH}, \mathrm{SH}, \mathrm{SM}$ and $\mathrm{AE}$ are the site investigators responsible for patient recruitment, data collection, endpoint assessment and judgment of image quality. All authors read and approved the final manuscript.

\section{Author details}

${ }^{1}$ Centre for Clinical Research, Unfallkrankenhaus Berlin Trauma Centre, Warener Str. 7, Berlin 12683, Germany. ${ }^{2}$ Department of Trauma and Orthopaedic Surgery, Unfallkrankenhaus Berlin Trauma Centre, Warener Str. 7, Berlin 12683, Germany. ${ }^{3}$ Department of Radiology, Unfallkrankenhaus Berlin Trauma Centre, Warener Str. 7, Berlin 12683, Germany. ${ }^{4}$ Julius Wolff Institute, Charité Medical University Centre, Augustenburger PI. 1, Berlin 13353, Germany.

Received: 26 December 2013 Accepted: 22 February 2014 Published: 3 March 2014 


\section{References}

1. 1. European Association for Injury Prevention and Safety Promotion: Injuries in the European Union. Report on Injury Statistics 2008-2010. Amsterdam: EuroSafe; 2013.

2. Mathers $C D$, Loncar D: Projections of global mortality and burden of disease from 2002 to 2030. PLoS Med 2006, 3:e442.

3. Murray CJ, Vos T, Lozano R, Naghavi M, Flaxman AD, Michaud C, Ezzati M, Shibuya K, Salomon JA, Abdalla S, Aboyans V, Abraham J, Ackerman I, Aggarwal R, Ahn SY, Ali MK, Alvarado M, Anderson HR, Anderson LM, Andrews KG, Atkinson C, Baddour LM, Bahalim AN, Barker-Collo S, Barrero LH, Bartels DH, Basáñez MG, Baxter A, Bell ML, Benjamin EJ, et al: Disability-adjusted life years (DALYs) for 291 diseases and injuries in 21 regions, 1990-2010: a systematic analysis for the Global Burden of Disease Study 2010. Lancet 2012, 380:2197-2223.

4. Peden M: UN General Assembly calls for decade of action for road safety. Inj Prev 2010, 16:213.

5. Celso B, Tepas J, Langland-Orban B, Pracht E, Papa L, Lottenberg L, Flint L: A systematic review and meta-analysis comparing outcome of severely injured patients treated in trauma centers following the establishment of trauma systems. J Trauma 2006, 60:371-378.

6. Davidson GH, Hamlat CA, Rivara FP, Koepsell TD, Jurkovich GJ, Arbabi S: Long-term survival of adult trauma patients. JAMA 2011, 305:1001-1007.

7. Mackenzie EJ, Rivara FP, Jurkovich GJ, Nathens AB, Frey KP, Egleston BL, Salkever DS, Scharfstein DO: A national evaluation of the effect of trauma-center care on mortality. N Engl J Med 2006, 354:366-378.

8. National Confidential Enquiry into Patient Outcome and Death: Trauma: Who Cares? 2007th edition. London: National Confidential Enquiry into Patient Outcome and Death; 2007.

9. Chen CW, Chu CM, Yu WY, Lou YT, Lin MR: Incidence rate and risk factors of missed injuries in major trauma patients. Accid Anal Prev 2011, 43:823-828.

10. Pfeifer R, Pape HC: Missed injuries in trauma patients: a literature review. Patient Saf Surg 2008, 2:20.

11. Kanz KG, Korner M, Linsenmaier U, Kay MV, Huber-Wagner SM, Kreimeier U, Pfeifer KJ, Reiser M, Mutschler W: Prioritätenorientiertes Schockraummanagement unter Integration des Mehrschichtspiralcomputertomographen. Unfallchirurg 2004, 107:937-944.

12. Salim A, Sangthong B, Martin M, Brown C, Plurad D, Demetriades D: Whole body imaging in blunt multisystem trauma patients without obvious signs of injury: results of a prospective study. Arch Surg 2006, 141:468-473.

13. Weninger P, Mauritz W, Fridrich P, Spitaler R, Figl M, Kern B, Hertz H: Emergency room management of patients with blunt major trauma: evaluation of the multislice computed tomography protocol exemplified by an urban trauma center. J Trauma 2007, 62:584-591.

14. Wurmb TE, Fruhwald P, Hopfner W, Roewer N, Brederlau J: Whole-body multislice computed tomography as the primary and sole diagnostic tool in patients with blunt trauma: searching for its appropriate indication. Am J Emerg Med 2007, 25:1057-1062.

15. Smith CM, Mason S: The use of whole-body CT for trauma patients: survey of UK emergency departments. Emerg Med J 2011, 29:630-634.

16. Huber-Wagner S, Lefering R, Qvick LM, Korner M, Kay MV, Pfeifer KJ, Reiser M, Mutschler W, Kanz KG, Working Group on Polytrauma of the German Trauma Society: Effect of whole-body CT during trauma resuscitation on survival: a retrospective, multicentre study. Lancet 2009, 373:1455-1461.

17. Huber-Wagner S, Biberthaler $\mathrm{P}$, Haberle $\mathrm{S}$, Wierer M, Dobritz M, Rummeny E, van Griensven M, Kanz KG, Lefering R, TraumaRegister DGU: Whole-body CT in haemodynamically unstable severely injured patients - a retrospective, multicentre study. PLoS One 2013, 8:e68880.

18. Stengel D, Ottersbach C, Matthes G, Weigeldt M, Grundei S, Rademacher G, Tittel A, Mutze S, Ekkernkamp A, Frank M, Schmucker U, Seifert J: Accuracy of single-pass whole-body computed tomography for detection of injuries in patients with major blunt trauma. CMAJ 2012, 2012:2012.

19. Asha S, Curtis KA, Grant N, Taylor C, Lo S, Smart R, Compagnoni K: Comparison of radiation exposure of trauma patients from diagnostic radiology procedures before and after the introduction of a panscan protocol. Emerg Med Australas 2012, 24:43-51.

20. Baumann BM, Chen EH, Mills AM, Glaspey L, Thompson NM, Jones MK, Farner MC: Patient perceptions of computed tomographic imaging and their understanding of radiation risk and exposure. Ann Emerg Med 2011, 58:1-7.

21. Brenner DJ, Hall EJ: Computed tomography-an increasing source of radiation exposure. $N$ Engl J Med 2007, 357:2277-2284.
22. Korley FK, Pham JC, Kirsch TD: Use of advanced radiology during visits to US emergency departments for injury-related conditions, 1998-2007. JAMA 2010, 304:1465-1471.

23. Sharma OP, Oswanski MF, Sidhu R, Krugh K, Culler AS, Stombaugh HA, Lauer SK: Radiation trends in trauma patients. Am Surg 2011, 77:193-197.

24. Snyder GE: Whole-body imaging in blunt multisystem trauma patients who were never examined. Ann Emerg Med 2008, 52:101-103.

25. Tien HC, Tremblay LN, Rizoli SB, Gelberg J, Spencer F, Caldwell C, Brenneman FD: Radiation exposure from diagnostic imaging in severely injured trauma patients. J Trauma 2007, 62:151-156.

26. Smith-Bindman R, Lipson J, Marcus R, Kim KP, Mahesh M, Gould R, Berrington de González A, Miglioretti DL: Radiation dose associated with common computed tomography examinations and the associated lifetime attributable risk of cancer. Arch Intern Med 2009, 169:2078-2086

27. Harrieder A, Geyer LL, Korner M, Deak Z, Wirth S, Reiser M, Linsenmaier U: Evaluation of radiation dose in 64-row whole-body CT of multiple injured patients compared to 4-row CT. Rofo 2012, 184:443-449.

28. Colli V, Mangini M, Strocchi S, Lumia D, Canì A, Boffano C, Cesarani F, Rizzi E, Ferrara RM, Andreis IA, Bruno J, Gozzi G, Cacciatori M, Ostinelli A, Vite C, Conte L, Fugazzola C: Performance assessment of four 64-slice computed tomographic devices for a typical clinical protocol. J Comput Assist Tomogr 2011, 35:57-64.

29. Thibault JB, Sauer KD, Bouman CA, Hsieh J: A three-dimensional statistical approach to improved image quality for multislice helical CT. Med Phys 2007, 34:4526-4544.

30. Silva AC, Lawder HJ, Hara A, Kujak J, Pavlicek W: Innovations in CT dose reduction strategy: application of the adaptive statistical iterative reconstruction algorithm. AJR Am J Roentgenol 2010, 194:191-199.

31. Kaplan DA: Iterative reconstruction in CT evolves for lower dose, increased clarity. 2012. http://www.diagnosticimaging.com/ct/iterativereconstruction-ct-evolves-lower-dose-increased-clarity.

32. Prakash P, Kalra MK, Kambadakone AK, Pien H, Hsieh J, Blake MA, Sahani DV: Reducing abdominal $\mathrm{CT}$ radiation dose with adaptive statistical iterative reconstruction technique. Invest Radiol 2010, 45:202-210.

33. Prakash P, Kalra MK, Ackman JB, Digumarthy SR, Hsieh J, Do S, Shepard JA, Gilman MD: Diffuse lung disease: $C T$ of the chest with adaptive statistical iterative reconstruction technique. Radiology 2010, 256:261-269.

34. Prakash P, Kalra MK, Digumarthy SR, Hsieh J, Pien H, Singh S, Gilman MD, Shepard JA: Radiation dose reduction with chest computed tomography using adaptive statistical iterative reconstruction technique: initial experience. J Comput Assist Tomogr 2010, 34:40-45.

35. Matsuda I, Hanaoka S, Akahane M, Sato J, Komatsu S, Inoh S, Kiryu S, Yoshioka N, Ino K, Ohtomo K: Adaptive statistical iterative reconstruction for volume-rendered computed tomography portovenography: improvement of image quality. Jpn J Radiol 2010, 28:700-706.

36. Ren Q, Dewan SK, Li M, Li J, Mao D, Wang Z, Hua Y: Comparison of adaptive statistical iterative and filtered back projection reconstruction techniques in brain CT. Eur J Radiol 2012, 81:2597-2601.

37. Hara AK, Paden RG, Silva AC, Kujak JL, Lawder HJ, Pavlicek W: Iterative reconstruction technique for reducing body radiation dose at CT: feasibility study. AJR Am J Roentgenol 2009, 193:764-771.

38. Noel PB, Fingerle AA, Renger B, Munzel D, Rummeny EJ, Dobritz M: Initial performance characterization of a clinical noise-suppressing reconstruction algorithm for MDCT. AJR Am J Roentgenol 2011, 197:1404-1409.

39. Hosch W, Stiller W, Mueller D, Gitsioudis G, Welzel J, Dadrich M, Buss SJ, Giannitsis E, Kauczor HU, Katus HA, Korosoglou G: Reduction of radiation exposure and improvement of image quality with BMl-adapted prospective cardiac computed tomography and iterative reconstruction. Eur J Radiol 2012, 81:3568-3576.

40. Funama Y, Taguchi K, Utsunomiya D, Oda S, Yanaga Y, Yamashita Y, Awai K: Combination of a low-tube-voltage technique with hybrid iterative reconstruction (iDose) algorithm at coronary computed tomographic angiography. J Comput Assist Tomogr 2011, 35:480-485.

41. Schoenhagen P, Bolen MA, Halliburton SS: Iterative CT reconstruction of aortic intramural hematoma. Circ J 2011, 75:1774-1776.

42. Noel PB, Fingerle AA, Renger B, Rummeny EJ, Doherty C: Clinical comparison study of a novel statistical iterative and filtered backprojection reconstruction. In Medical Imaging 2011: Physics of Medical Imaging. Proceedings of SPIE 7961. Edited by Pelc NJ, Samei E, Nishikawa RM. 2011. 
43. Philips Healthcare: iDose4 Iterative Reconstruction Technique. Breakthrough in image Quality and Dose Reduction With the 4th Generation of Reconstruction. Eindhoven: Philips Healthcare; 2011.

44. Maxfield MW, Schuster KM, McGillicuddy EA, Young CJ, Ghita M, Bokhari SA, Oliva IB, Brink JA, Davis KA: Impact of adaptive statistical iterative reconstruction on radiation dose in evaluation of trauma patients. J Trauma Acute Care Surg 2012, 73:1406-1411.

45. Deutsche Gesellschaft für Unfallchirurgie: S3-Leitlinie "Polytrauma/ Schwerverletzten-Behandlung" (AWMF Register-Nr. 012/019). DGU Mitteil Nachr 2011, 33:1-188.

doi:10.1186/1757-7241-22-15

Cite this article as: Stengel et al:: Dose reduction in whole-body computed tomography of multiple injuries (DoReMI): protocol for a prospective cohort study. Scandinavian Journal of Trauma, Resuscitation and Emergency Medicine 2014 22:15.

\section{Submit your next manuscript to BioMed Central and take full advantage of:}

- Convenient online submission

- Thorough peer review

- No space constraints or color figure charges

- Immediate publication on acceptance

- Inclusion in PubMed, CAS, Scopus and Google Scholar

- Research which is freely available for redistribution 